\title{
Two-pole Analysis of Interconnection Trees *
}

\author{
Andrew B. Kahng and Sudhakar Muddu \\ UCLA Computer Science Department, Los Angeles, CA 90024-1596 USA
}

\begin{abstract}
We address the two-pole simulation of interconnect trees via the moment matching technique. We simulate the interconnect network by modeling the distributed lines with non-uniform lumped segments and using the two-pole methodology. To this end, we derive new nonuniform equivalent circuits which match the general distributed line transfer function up to the second term. Using the recursive equation for the admittance of a tree, we give the exact expressions for the first and second moments of the transfer function of the interconnect tree. Our results show that delay estimates using our method are within $13 \%$ of SPICE-computed delays. As routing trees become bigger and interconnection lines become longer, e.g., in MCM design, our approach has advantages in both accuracy and simulation complexity. significant.
\end{abstract}

\section{Introduction}

As feature sizes decrease and operating frequencies increase, interconnect delays come to dominate gate delays. Thus, interconnects are a major factor in the performance of high-speed integrated circuit, multichip and system-level designs. Various techniques have been proposed for the simulation of interconnects. Direct simulation techniques such as SPICE give the most accurate insight into arbitrary interconnect structures, but are computationally expensive. Asymptotic waveform evaluation (AWE) [PR90] simulates transmission line networks based on moment computations: individual interconnects are modeled using distributed 2-port parameters and the node voltages are recursively calculated by solving the circuit equations.

Faster techniques such as the two-pole approach [Hor84, ZSTGC94] have been used to calculate the response using the first and second moments. Traditionally, with these and previous approaches interconnects are modeled using uniform lumped $R C$ and $R L C$ segments. However, as interconnect lengths and operating frequencies increase, such uniform models can lead to errors, typically because only a few uniform lumped segments are computationally reasonable in modeling the interconnects, and moments are not captured exactly. ${ }^{1}$ In [KM94] the two-pole response was obtained using

\footnotetext{
*This work was supported by NSF MIP-9257982.

${ }^{1}$ Also, Zhou et al. [ZSTGC94] calculate the response using an empirical relationship for the moment computation and approximate the off-path impedance as the sum of total subtree capacitance. This error in the second moment and pole computation becomes significant as the size of off-path subtrees increases, as in MCM interconnects.
}

non-uniform segment models which exactly match the first two or three moments of the transfer function of an open-ended distributed transmission line. The resulting response and delay estimates were found to be more accurate than those of previous approximate methods in [Hor84, ZSTGC94].

In this paper, we make the following contributions. First, we propose new accurate non-uniform $R L C$ segment models for general distributed interconnect lines. Second, we present exact expressions for the first and second moments of the interconnect tree transfer function, via a recursive expression for the admittance of a subtree which captures off-path subtree admittance accurately. We then simulate interconnect trees using the non-uniform segment models and the two-pole methodology [Hor84, ZSTGC94]. For estimation of 90\% threshold delay, our method is within $13 \%$ of the SPICE-computed delays for a range of interconnect parameter values and routing structures. While we use two-pole techniques for response calculations, the new segment models can be incorporated within $R L C$ model libraries for other circuit simulators. Improvements in accuracy and savings in simulation costs can be significant in the design of high-speed systems.

\section{$2 \quad$ Lumped Segment Models}

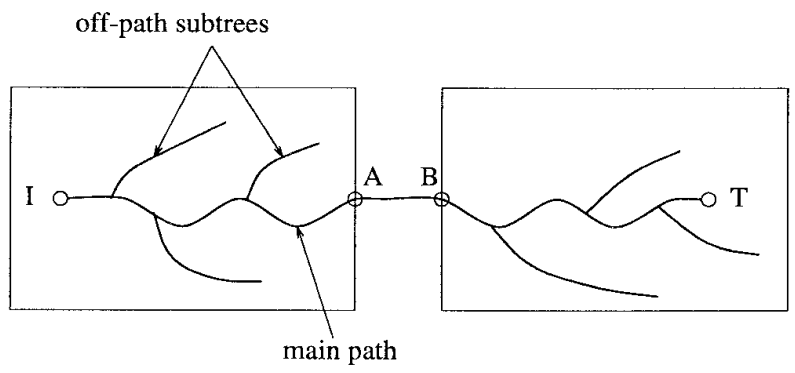

Figure 1: The main path of the routing tree between source $I$ and load $T$.

To model distributed $R L C$ and $R C$ lines, uniform $\mathbf{L}$, $\mathbf{T}$ or $\boldsymbol{\Pi}$ models have traditionally been used. The accuracy of these models is highly dependent on the number of segments used for each distributed line. As the number of segments tends to infinity, the $\mathbf{L}$ type model approaches the $R L C$ distributed line model [KM93]. Sakurai [Sak83] showed that for both the $\mathbf{T}$ and $\boldsymbol{\Pi}$ models, as the number of segments tends to infinity the equivalent circuit transfer function also converges to the distributed $R L C$ transfer function. In [Raj74, KM94] non-uniform 
segments for interconnect lines are developed by comparing with the open-ended transfer function of the transmission line. Using only a few non-uniform segments to model distributed $R C / R L C$ lines, the coefficients of the open-ended transfer function are matched accurately. A comparison of various lumped models approximating the distributed $R L C$ line is given in [KM94]. Gerzberg [Ger79] surveyed different non-uniform models and proposed a model in which the segment $R C$ values are in geometric progression (the "Uniform Distributed RC" (URC) line model in SPICE is derived from Gerzberg's model). ${ }^{2}$ We now develop non-uniform equivalent circuit models for a general interconnect line with source and load impedances.

\subsection{Distributed $R L C$ Line Model}

Consider the interconnect line $A B$ shown in the tree example (Figure 1). To model this interconnect line, we consider the source resistance and inductance, which are respectively equal to the resistance and inductance of the main path from the source to the interconnect line. We approximate the load impedance by the total subtree capacitance at the end of the line. A representation of the interconnect line $A B$ with source and load impedances is shown in Figure 2. The $A B C D$ parameters of a distributed $R L C$ transmission line (Figure 2) are

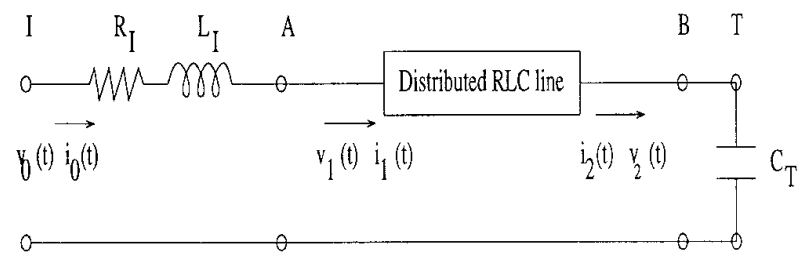

Figure 2: 2-port model of a distributed $R L C$ line with source impedance $Z_{I}$.

$$
\left(\begin{array}{c}
V_{1}(s) \\
I_{1}(s)
\end{array}\right)=\left(\begin{array}{cc}
\cosh (\theta h) & Z_{0} \sinh (\theta h) \\
\frac{1}{Z_{0}} \sinh (\theta h) & \cosh (\theta h)
\end{array}\right)\left(\begin{array}{c}
V_{2}(s) \\
I_{2}(s)
\end{array}\right)
$$

where $\theta=\sqrt{(r+s l) s c}, h=$ length of the line, and $r=\frac{R}{h}, l=\frac{L}{h}$ and $c=\frac{C}{h}$ are the resistance, inductance and capacitance per unit length. By modeling the interconnect line using the 2-port parameters the transfer function between nodes $I$ and $T$ is given by [KM94]:

$H(s)=\frac{V_{I}(s)}{V_{T}(s)}=\frac{1}{\cosh (\theta h)\left(1+\frac{Z_{I}}{Z_{T}}\right)+\sinh (\theta h)\left(\frac{Z_{I}}{Z_{0}}+\frac{Z_{0}}{Z_{T}}\right)}$

where $Z_{T}=\frac{1}{s C_{T}}, Z_{I}=R_{I}+s L_{I}, Z_{0}=\sqrt{\frac{R+s L}{s C}}$ and $\theta h=\sqrt{(R+s L) s C}$. Expanding cosh and sinh as infinite series and collecting terms up to the coefficient of $s^{2}$ in

\footnotetext{
${ }^{2}$ The concept of non-uniform equivalent circuits has also been employed in other areas, e.g., O'Brien et al. [OS89] and Gopal et al. [GNP91] obtain a non-uniform segment model for driving-point impedance at the gate output using moment matching techniques.
}

the denominator, we get $^{3}$

$$
H(s)=\frac{1}{1+s b_{1}^{I T}+s^{2} b_{2}^{I T}+\ldots}
$$

where

$$
\begin{aligned}
b_{1}^{I T}= & R_{I} C+R_{I} C_{T}+\frac{R C}{2}+R C_{T} \\
b_{2}^{I T}= & \frac{R_{I} R C^{2}}{6}+\frac{R_{I} R C C_{T}}{2}+\frac{(R C)^{2}}{24}+\frac{R^{2} C C_{T}}{6} \\
& +L_{I} C+L_{I} C_{T}+\frac{L C}{2}+L C_{T}
\end{aligned}
$$

\subsection{Computation of Equivalent Circuit Models}

$\begin{array}{llllllllllllllllllll}Y_{1} & R_{1} & L_{1} & V_{N-1} & R_{N} & L_{N} & V_{N} & R_{N-1} & L_{N-1} & Y_{N-1} & V_{2} & R_{1} & L_{1} & Y_{1} & B & & N\end{array}$

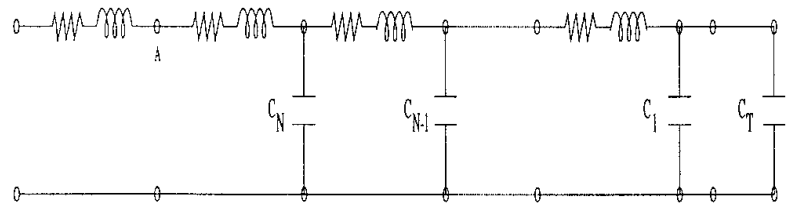

Figure 3: $N$-segment distributed $R L C$ transmission line model with source resistance $R_{I}$, source inductance $L_{I}$ and load capacitance $C_{T}$.

In [Raj74, KM94] non-uniform equivalent circuits for $R L C$ lines were developed by assuming an open-ended line. Under this assumption two non-uniform $R L C$ segments are sufficient to match the transfer function and the input impedance of the line up to the coefficient of $s^{2}$. Here, to derive non-uniform equivalent circuits for a distributed line with general load and source impedances, we need three non-uniform $R L C$ segments to match up to the coefficient of $s^{2}$ in the transfer function. Consider the interconnect line $A B$ of Figure 1 , represented with $N R L C$ segments as shown in Figure 3 . To match the transfer function coefficient up to the required accuracy, we use the following expression for the $k^{t h}$ coefficient $b_{k}$ from [KM93]:

$$
b_{k}^{N+1}=R_{N} \sum_{j=1}^{N} C_{j} b_{k-1}^{j}+L_{N} \sum_{j=1}^{N} C_{j} b_{k-2}^{j}+b_{k}^{N}
$$

From this recursive equation the $k^{\text {th }}$ coefficient of the transfer function between nodes $I$ and $T$ can be computed as

$$
b_{k}^{I T}=R_{I}\left(\sum_{j=1}^{N} C_{j} b_{k-1}^{j}+C_{T} b_{k-1}^{T}\right)
$$

\footnotetext{
${ }^{3}$ Similarly, the transfer function of the open-ended distributed $R L C$ line is given by

$$
H(s)=\frac{1}{1+\frac{R C}{2} s+\left(\frac{(R C)^{2}}{24}+\frac{R C}{2}\right) s^{2}+\left(\frac{(R C)^{3}}{720}+\frac{R L C^{2}}{12}\right) s^{3}+\ldots}
$$
}




$$
\begin{aligned}
& +L_{I}\left(\sum_{j=1}^{N} C_{j} b_{k-2}^{j}+C_{T} b_{k-2}^{T}\right) \\
& +\sum_{j=1}^{N} C_{j} b_{k-1}^{j} \sum_{i=j}^{N} R_{i}+C_{T} b_{k-1}^{j} \sum_{i=1}^{N} R_{i} \\
& +\sum_{j=1}^{N} C_{j} b_{k-2}^{j} \sum_{i=j}^{N} L_{i}+C_{T} b_{k-2}^{j} \sum_{i=1}^{N} L_{i}
\end{aligned}
$$

where $b_{0}=1$ and $b_{-1}=0$. Therefore,

$$
\begin{aligned}
b_{1}^{I T}= & R_{I}\left(\sum_{j=1}^{N} C_{j}+C_{T}\right)+\left(\sum_{j=1}^{N} C_{j} \sum_{i=j}^{N} R_{i}\right) \\
& +C_{T}\left(\sum_{i=1}^{N} R_{i}\right) \\
b_{2}^{I T}= & R_{I}\left(\sum_{j=1}^{N} C_{j} b_{1}^{j}+C_{T} b_{1}^{T}\right)+L_{I}\left(\sum_{j=1}^{N} C_{j}+C_{T}\right) \\
& +\sum_{j=1}^{N} C_{j} b_{1}^{j} \sum_{i=j}^{N} R_{i}+C_{T} b_{1}^{T} \sum_{i=1}^{N} R_{i} \\
& +\sum_{j=1}^{N} C_{j} \sum_{i=j}^{N} L_{i}+C_{T} \sum_{i=1}^{N} L_{i}
\end{aligned}
$$

For a uniformly distributed segment model with $R_{i}=\frac{R}{N}$, $L_{i}=\frac{L}{N}$ and $C_{i}=\frac{C}{N}$, the transfer function coefficients from Equation (3) in the limit as $N \rightarrow \infty$ are the same as those given in Equation (2).

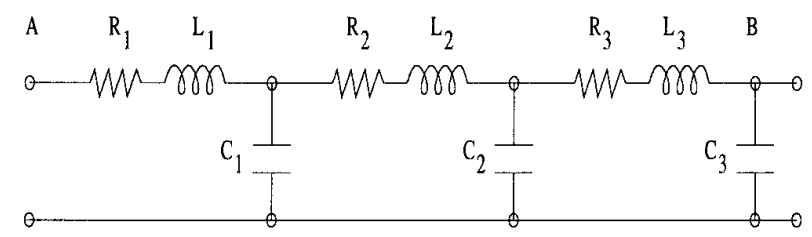

Figure 4: Non-uniform three $\mathrm{L}$ segment model for a distributed $R L C$ line with source and load.

We obtain our non-uniform equivalent circuit parameters by computing the coefficients using Equation (3) and matching with the distributed transfer function coefficients given by Equation (2). From Equation (3) we can see that the constraints imposed on the resistance parameters are the same as the constraints on the inductance parameters, i.e., for any non-uniform $R L C$ equivalent circuits the resistances and inductances are identically distributed:

$$
R_{i}=L_{i} \quad \forall i
$$

Therefore, we need derive constraints only for resistance and capacitance parameters of the equivalent circuit. Observe that there are four different terms with resistance and capacitance values in each coefficient of $s^{k}$.
These are the source resistance $\left(R_{I}\right)$ term, the source resistance and load capacitance $\left(R_{I} C_{T}\right)$ term, the line resistance and capacitance $(R C)$ term, and the line resistance and load capacitance $\left(R C_{T}\right)$ term. However, the source resistance and load capacitance $\left(R_{I} C_{T}\right)$ term in the coefficient of $s$ does not yield any constraints, so there are a total of $4 k-1$ constraints in matching up to $k$ coefficients in the equivalent circuit. The number of non-uniform $\mathbf{L}$ or $\Pi \mathbf{I}$ segments required to match these constraints is at least $2 k-1$ [KM93]. In particular, to derive equivalent circuits which match up to the coefficient of $s^{2}$ we need to satisfy 7 constraints; this can be achieved by using $3 \mathbf{L}$ or $\boldsymbol{\Pi}$ segments. (Note that with $\mathbf{L}$ segments we get an overspecified system of equations.)

Our methodology uses numerical search to solve for the equivalent circuit parameters. The equivalent $\mathbf{L}$ circuit parameters (Figure 4) for matching up to the second moment are given by

$$
\begin{array}{rll}
R_{1}=0.20 R, & R_{2}=0.40 R, & R_{3}=0.40 R \\
L_{1}=0.20 L, & L_{2}=0.40 L, & L_{3}=0.40 L \\
C_{1}=0.42 C, & C_{2}=0.41 C, & C_{3}=0.17 C
\end{array}
$$

Similarly, the equivalent $\Pi$ circuit parameters (Figure 5) are obtained as

$$
\begin{array}{cll}
R_{1}=0.34 R, \quad R_{2}=0.32 R, & R_{3}=0.34 R \\
L_{1}=0.34 L, \quad L_{2}=0.32 L, & L_{3}=0.34 L \\
C_{0}=0.15 C, \quad C_{1}=0.35 C, \quad C_{2}=0.35 C, \quad C_{3}=0.15 C
\end{array}
$$

Note that the $\boldsymbol{\Pi}$ circuit parameters are symmetrical, in contrast to the $\mathbf{L}$ circuit model. The coefficients of the transfer function using the $\boldsymbol{\Pi}$ circuit parameters are

$$
\begin{aligned}
b_{1}^{I B}= & R_{I} C+R_{I} C_{T}+\frac{R C}{2}+R C_{T} \\
b_{2}^{I B}= & \frac{R_{I} R C^{2}}{6.0}+\frac{R_{I} R C C_{T}}{2}+\frac{(R C)^{2}}{27.11}+\frac{R^{2} C C_{T}}{6.37} \\
& +L_{I} C+L_{I} C_{T}+\frac{L C}{2}+L C_{T}
\end{aligned}
$$

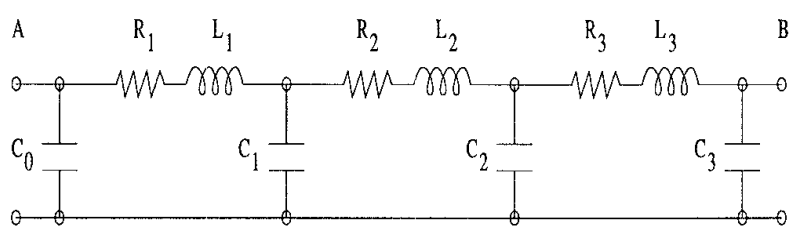

Figure 5: Non-uniform three II segment model for a distributed $R L C$ line with source and load.

These new equivalent circuit models should be contrasted with previous models in [KM94], which were derived under the open-ended assumption and by matching the transfer function coefficients up to $s^{3}$. For instance, our previous three $\mathbf{L}$ segment model for the open-ended line had parameters

$$
R_{1}=0.30 R, \quad R_{2}=0.20 R, \quad R_{3}=0.50 R
$$




$$
\begin{array}{lll}
L_{1}=0.30 L, & L_{2}=0.20 L, & L_{3}=0.50 L \\
C_{1}=0.40 C, & C_{2}=0.44 C, & C_{3}=0.16 C
\end{array}
$$

Because non-uniform equivalent circuits match the distributed line moments accurately, using such equivalent circuits in a two-pole or higher-order approximation of the transfer function will achieve a more accurate voltage response than simply using uniform segment models (e.g., as in [ZSTGC94]). For large routing trees such as in MCM substrates, the use of non-uniform equivalent circuit models will reduce computation time significantly. The non-uniform equivalent circuits can also be employed in place of the lumped $\mathbf{T}$ and $\boldsymbol{\Pi}$ models that are traditionally used for clock skew minimization and other performance-driven routing applications.

\section{Interconnect Tree Analysis}

We now describe the approach for calculating the response at a given sink of a general interconnection tree. Previous two-pole methods calculate the two dominant poles of the transfer function from the first and second moments by modeling each distributed line separately with uniform equivalent circuits [Hor84, GZ93]. To improve the accuracy of the response, Zhou et al. [ZSTGC94] consider a special polynomial function that describes the poles by heuristically incorporating a model proposed by [ZPK91]. To further improve accuracy, the authors of [ZSTGC94] model each tree branch by many (uniform) shorter segments. This method may not be practical for trees with long wire segments (e.g., on an MCM substrate, where a lossy transmission line model is most relevant).

We propose to compute the poles of the transfer function by modeling each distributed line with the above non-uniform equivalent circuits. In computing the moments of the tree, we represent the off-path subtrees by their respective admittance values instead of approximating by total subtree capacitance. Thus, we must derive the exact expressions for the first and second moments in terms of the lumped segment parameters used to model the distributed lines.

\subsection{Tree Moment Computations}

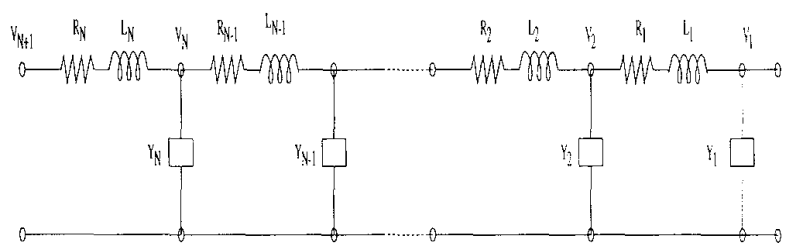

Figure 6: Representation of the main path in the tree, where each distributed line is modeled using $R L C$ segments. $Y_{i}$ indicates the off-path subtree admittance.

Consider the main path between the source and sink of interest, and replace each subtree by its respective admittance. To calculate the response at the sink we use an approach similar to that of Gao et al. [GZ93]. Figure 6 shows an example of a main path where each branch in the tree is replaced by a single $R L C$ segment, and subtrees are replaced by their respective admittances.
The node $V_{N+1}$ indicates the source, and $V_{1}$ indicates the sink of interest. At any node $i$ the admittance $Y_{i}$ is equal to the capacitance at the node $i$ if there is no subtree at node $i$. If there is a subtree at node $i$ then $Y_{i}$ is equal to the sum of the subtree admittance and the admittance of the capacitance of the equivalent circuit, i.e.,
$Y_{i}=s C_{i}$
$=s C_{i}+Y_{s u b t r e e i}$ if node $i$ has off-path subtree

For this equivalent circuit the input voltage can be written as

$$
V_{N+1}(s)=\left(R_{N}+s L_{N}\right)\left(\sum_{j=1}^{N} Y_{j}(s) V_{j}(s)\right)+V_{N}(s)
$$

where $Y_{j}(s)=s Y_{1, j}+s^{2} Y_{2, j}+\ldots$, with $Y_{1, j}$ and $Y_{2, j}$ being the coefficients of $s$ and $s^{2}$ of the subtree admittance. Expressing $V_{N+1}(s)$ as a series expansion of $s, V_{N+1}(s)=$ $V_{1}(s)\left(1+b_{1}^{N+1} s+b_{2}^{N+1} s^{2}+b_{3}^{N+1} s^{3}+\ldots\right)$. The general expression for the transfer function coefficient of $s^{k}$ is

$$
\begin{aligned}
b_{k}^{N+1}= & R_{N} \sum_{l=1}^{k}\left(\sum_{j=1}^{N} Y_{l, j} b_{k-l}^{j}\right) \\
& +L_{N} \sum_{l=1}^{k-1}\left(\sum_{j=1}^{N} Y_{l, j} b_{k-1-l}^{j}\right)+b_{k}^{N}
\end{aligned}
$$

Thus, we have

$$
\begin{aligned}
b_{1}^{N+1} & =R_{N} \sum_{j=1}^{N} Y_{1, j}+b_{1}^{N}=\sum_{i=1}^{N} R_{i} \sum_{j=1}^{i} Y_{1, j} \\
& =\sum_{j=1}^{N} Y_{1, j} \sum_{i=j}^{N} R_{i}
\end{aligned}
$$

and similarly $b_{2}^{N+1}$ is given by,

$$
\begin{aligned}
b_{2}^{N+1}= & R_{N} \sum_{j=1}^{N} Y_{1, j} b_{1}^{j}+L_{N} \sum_{j=1}^{N} Y_{1, j} \\
& +R_{N} \sum_{j=1}^{N} Y_{2, j}+b_{2}^{N} \\
= & \sum_{j=2}^{N} Y_{1, j} \sum_{l=j}^{N} R_{l} \sum_{i=1}^{j-1} Y_{1, j} \sum_{d=i}^{j-1} R_{d} \\
& +\sum_{j=1}^{N} Y_{1, j} \sum_{l=j}^{N} L_{l}+\sum_{j=1}^{N} Y_{2, j} \sum_{l=j}^{N} R_{l}
\end{aligned}
$$

We thus obtain a general expression for coefficients of the transfer function in terms of parameters of the main path and the subtree admittance coefficients. The moments of 
the transfer function can be calculated from the transfer function coefficients using the recursive equation [KM93]:

$$
M_{k}=(-1)^{k+1} \sum_{i=1}^{k} b_{i} M_{k-i}
$$

The first and second moment expressions are given by:

$$
\begin{aligned}
M_{1}= & b_{1}=\sum_{j=1}^{N} Y_{1, j} \sum_{i=j}^{N} R_{i} \\
M_{2}= & b_{1}^{2}-b_{2}=\left(\sum_{j=1}^{N} Y_{1, j} \sum_{i=j}^{N} R_{i}\right)^{2}-\sum_{j=1}^{N} Y_{2, j} \sum_{l=j}^{N} R_{l} \\
& -\sum_{j=2}^{N} Y_{j}^{1} \sum_{l=j}^{N} R_{l} \sum_{i=1}^{j-1} Y_{i}^{1} \sum_{d=i}^{j-1} R_{d}-\sum_{j=1}^{N} C_{j} \sum_{l=j}^{N} L_{l}
\end{aligned}
$$

\subsection{Computation of Subtree Admittance}

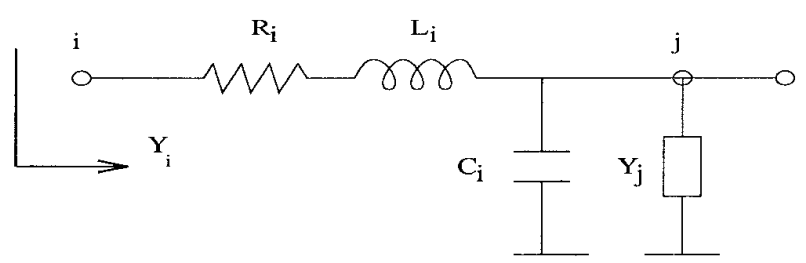

Figure 7: A single $R L C$ segment between nodes $i$ and $j$.

O'Brien et al. [OS90] gave a recursive computation for calculating the admittance coefficients for RC trees. In their method, admittance coefficients are obtained by considering each element in the tree in an iterative fashion. Later Sriram et al. [SK93] obtain an expression for the coefficient of $s$ and $s^{2}$ for admittance for a series section of $R L C$ segments, but their coefficient of $s^{2}$ in the subtree admittance is different from what we derived below. In this subsection, we obtain the expression for the first $Y_{1, i}$ and second $Y_{2, i}$ terms of the admittance for a subtree.

In general, the admittance at node $i$ can be expressed in terms of the admittance at node $j$ as shown in Figure 7 . In the figure, $Y_{j}$ indicates the admittance of the subtree rooted at node $j$.

$$
\begin{aligned}
Y_{i} & =\frac{1}{R_{i}+s L_{i}+\frac{1}{\left(Y_{j}+s C_{i}\right)}} \\
& =\left(Y_{j}+s C_{i}\right)-\left(Y_{j}+s C_{i}\right)^{2} R_{i}-s L_{i}\left(Y_{j}+s C_{i}\right)^{2}+\ldots
\end{aligned}
$$

Using the above recursive equation, the admittance of the off-path subtrees can be computed. By writing the admittance $Y_{j}$ at node $j$ as an infinite series, the admittance at node $i$ is given by

$$
Y_{i}=s\left(Y_{1, j}+C_{i}\right)-s^{2}\left(Y_{2, j}+R_{i}\left(Y_{1, j}+C_{i}\right)^{2}\right)+\ldots
$$

and the $s$ and $s^{2}$ coefficients of the admittance are seen to be

$$
\begin{aligned}
& Y_{1, i}=C_{i}+Y_{1, j} \\
& Y_{2, i}=-R_{i}\left(Y_{1, j}+C_{i}\right)^{2}+Y_{2, j}
\end{aligned}
$$

By induction, the admittance coefficients $s$ and $s^{2}$ for $N$ $R L C$ segments connected in series (Figure 3 ) are given by

$$
Y_{1, N+1}=\sum_{j=1}^{N} C_{j} \quad ; \quad Y_{2, N+1}=-\sum_{i=1}^{N} R_{i}\left(\sum_{\forall j \in S_{T(i)}} C_{j}\right)^{2}
$$

where $S_{T(i)}$ denotes the set of nodes in subtree $T(i)$ rooted at node $i$. From Equation (6), we may compute the coefficients of $s$ and $s^{2}$ of the admittance, which are then used in the first and second moment calculations in Equation (5).

\section{Experimental Results}

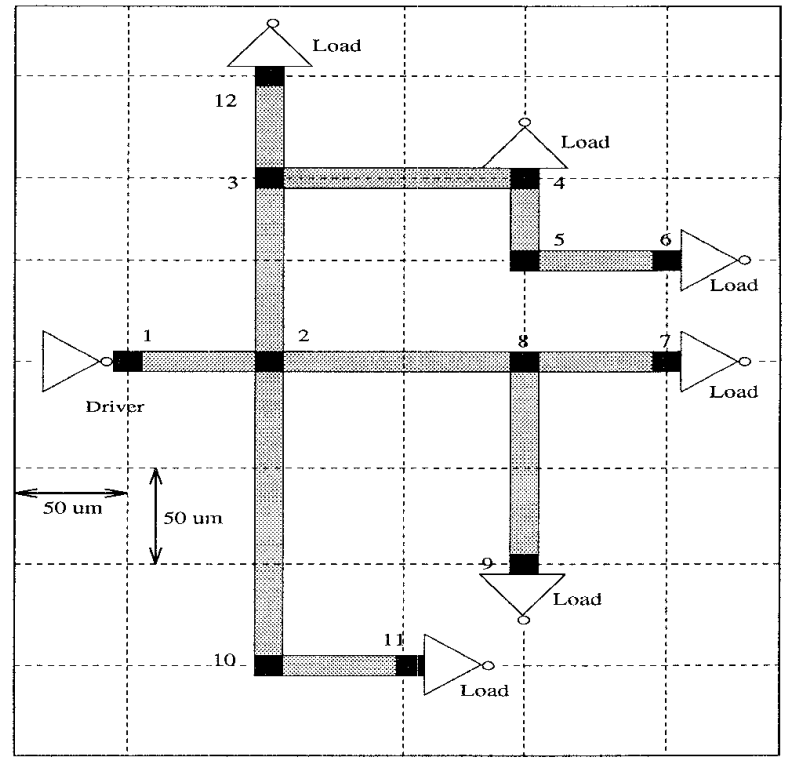

Figure 8: A tree interconnection layout studied in [ZSTGC92].

We conclude with a practical demonstration of the effect of non-uniform equivalent circuit models and the two-pole simulation technique, using exact admittance calculations. We consider the tree interconnection layout given in Figure 8 . We calculate the $90 \%$ threshold delay at node 6 using both SPICE and the two-pole methodology described above. The SPICE simulation of the tree was performed using the built-in LTRA (Lossy TRAnsmission line) model for each tree segment. In our twopole method we replaced each segment using the nonuniform II segment model (Figure 5) and calculated the response using the first and second moment computation. We calculated the delay values for various interconnect parameters, driver resistances and grid sizes as 


\begin{tabular}{||c|c|c|c|c|c||}
\hline $\begin{array}{c}\text { Driver } \\
\text { resistance } \\
\Omega\end{array}$ & $\begin{array}{c}\text { Interconnect } \\
\text { parameters } \\
/ \mu m\end{array}$ & $\begin{array}{c}\text { Load } \\
\text { capacitance } \\
p F\end{array}$ & $\begin{array}{c}\text { Grid } \\
\text { size } \\
\mu m\end{array}$ & $\begin{array}{c}\text { SPICE } \\
\text { delay } \\
p s\end{array}$ & $\begin{array}{c}\text { Two-pole } \\
\text { delay } \\
p s\end{array}$ \\
\hline \hline 100 & $\begin{array}{c}R=0.015 \Omega \\
C=0.176 f F \\
L=0.246 p H\end{array}$ & 2.0 & 50 & 54 & 56 \\
\hline 10 & $\begin{array}{c}R=0.015 \Omega \\
C=0.176 f F \\
L=0.24 p H\end{array}$ \\
\hline 10 & $\begin{array}{c}R=0.015 \Omega \\
C=0.176 f F \\
L=24.6 p H\end{array}$ & 2.0 & 500 & 345 & 391 \\
\hline 10 & $\begin{array}{c}R=0.015 \Omega \\
C=17.6 \mathrm{fF} \\
L=0.24 \mathrm{pH}\end{array}$ & 2.0 & 50 & 689 & 719 \\
\hline
\end{tabular}

Table 1: The 90\% threshold delay values at node 6 using both SPICE and the two-pole methodology. Note that these delay values indicate the rise-time delay only.

shown in Table 1. The delay values shown in the table indicate the rise-time delay; total delay can be computed by adding propagation delay to the rise-time delay. The two-pole delays are within $13 \%$ of the SPICE-computed delays.

As the wire length increases, the difference between these models becomes much more significant. For highspeed systems or MCM layout applications where the wire lengths become very large, our approach will allow improved accuracy and efficiency when compared with previous two-pole methods.

\section{References}

[E48] W. C. Elmore, "The Transient. Response of Damped Linear Networks with Particular Regard to Wideband Amplifiers", J. App. Physics 19, Jan. 1948, pp. 55-63.

[Ger79] L. Gerzberg, "Monolithic Power-Spectrum Centroid Detector", PhD Thesis, Stanford Univ., May 1979.

[GNP91] N. Gopal, D. P. Neikirk and I. T. Pillage, "Evaluating RC-Interconnect Using Moment-Matching Approximations", Proc. IEEE ICCAD, June 1991, pp. 74-77.

[GZ93] D. S. Gao and D. Zhou, "Propagation Delay in RLC Interconnection Networks", Proc. Intl. Symposium on Circuits and Systems, May 1993, pp. 2125-2128.

[Hor84] M. A. Horowitz, "Timing Models for MOS Circuits", PhD Thesis, Stanford Univ., Jan. 1984.

[KM93] A. B. Kahng and S. Muddu, "Delay Estimation of Trees Using Two-pole Method and Optimal Equivalent Circuits", UCLA CS Dept. TR-930035, October 1993, with addendum 1994.

[KM94] A. B. Kahng and S. Muddu, "Optimal Equivalent Circuits for Interconnect Delay Calculations Using Moments", Proc. ACM/IEEE European Design Automation Conf., September 1994, pp. 164-169.

[OS89] P. R. O'Brien and T. L. Savarino, "Modeling the Driving-Point Characteristic of Resistive Interconnect for Accurate Delay Estimation", Proc. IEEE ICCAD, 1989, pp. 512-515.
[OS90] P. R. O'Brien and T. L. Savarino, "Efficient On-Chip Delay Estimation for Leaky Models of Multiple-Source Net", IEEE Custom Integrated Circuits Conf., 1990.

[PR90] L. T. Pillage and R. A. Rohrer, "Asymptotic Waveform Evaluation for Timing Analysis", IEEE Trans. CAD, Apr. 1990, pp.352-366.

[Raj74] Y. V. Rajput, "Modelling Distributed RC Lines for the Transient Analysis of Complex Networks", Int. J. Electronics, 1974, pp. 709-717.

[Sak83] T. Sakurai, "Approximation of Wiring Delay in MOSFET LSI", IEEE J. Solid-State Circuits, Aug. 1983, pp. 418-426.

[SK93] M. Sriram and S. M. Kang, "Performance driven MCM Routing Using a Second Order RLC Tree Delay Model", Proc. IEEE Intl. Con. Wafer Scale Integration, Jan. 1993, pp. 262-267.

[ZPK91] D. Zhou, F. P. Preparata and S. M. Kang, "Interconnection Delay in Very High-Speed VLSI", IEEE Trans. on Circuits and Systems 38(7), July 1991, pp. 779-790.

[ZSTGC94] D. Zhou, S. Su, F. Tsui, D. S. Gao and J. S. Cong, "A Simplified Synthesis of Transmission Lines with A Tree Structure", Intl. Journal of Analog Integrated Circuits and Signal Processing, Jan. 1994, pp. 19-30. 